\title{
Reviews
}

Journal of Direct, Data and Digital Marketing Practice (2012) 14, 77-80. doi:10.1057/dddmp.2012.12

\section{Marketing Analytics 2011 - Conference report}

The 2011 edition of Henry Stewart's long-running series of Marketing Analytics conferences was a 2-day event held in London last December. There were two overall themes, corresponding broadly to the separate days — those advanced analytics techniques that may be applied to any business problems where appropriate data exist, and the new generation of methods that have been developed for the online world.

As in previous years, the event was primarily designed as a "userfriendly' briefing on the wide range of advanced analytics methods employed in marketing, illustrating their applications using case study examples. This report briefly summarizes the wealth of talks that took place over the two days.

\section{Advanced analytics and data mining}

\section{Factor analysis}

\section{Cluster analysis}

\section{Day 1 - Briefing on advanced analytics techniques}

The first day was chaired by Andrew Dellbridge (Nationwide Building Society), who introduced the proceedings by challenging delegates to think about how they will embed analytics into their businesses.

Barry Leventhal (BarryAnalytics) presented an overview of the advanced analytics landscape and suggested five key themes for delegates to bear in mind - such as the critical dependence on data and the importance of data integration. The presentation included a live demonstration of a tool developed by NAG Software, which applied different data mining techniques to a set of data and compared their performance.

Dave Walter (dunnhumby) gave a thorough explanation of factor analysis, and its 'sister technique' principal components analysis, for data reduction and identifying underlying dimensions in data. These methods can sometimes seem 'dry' and 'full of jargon'; however, Dave brought them to life by illustrating their usefulness for analysing Tesco's grocery shopping data.

Corrine Moy (GfK NOP) discussed the use of cluster analysis and other statistical techniques for segmentation. Corrine stressed the importance of choosing the right approach, before embarking on a segmentation project, by answering a series of key questions about why and how the segmentation will be used. After explaining the main clustering techniques, Corrine closed by explaining how to select a good segmentation solution. 
Decision trees

Advanced application of
regression analysis

Advanced data visualization

Analysing the social networks of customers

Web analytics

\section{Online marketing analytics}

\section{Social media monitoring}

Tom Breur (XLNT Consulting) presented the use of Decision Trees to 'divide and conquer' large data sets. Tom explained the principles of tree analysis, the main algorithms in use and a series of highly practical points on interpreting the outputs.

Tony Lovick (Towers Watson) discussed the application of regression models to insurance data, and explained why improved techniques are required for more accurate pricing. Tony introduced the Case Deleted Deviance, as a measure of variability for models, and showed how this can be used to reduce the 'noise' in model estimates - resulting in a gain in profit margins.

Peter Furness (Peter Furness Limited) explained that the aim of 'Advanced Data Visualization' is to summarize large amounts of data clearly in a visual display, based on established principles of graphical excellence. Peter presented a large number of examples, including 'mash-ups' of data overlaid onto geographical maps.

Judy Bayer (Teradata) introduced Social Network Analysis (SNA) as a technique for measuring relationships between individuals, such as customers of a phone network. Judy then presented a number of business applications, including churn management, cross-sell, viral marketing and family identification, and discussed the extension of SNA to Social Media Analysis. Judy concluded with a case study example from an East European mobile phone company.

Garry Lee (RedEye) gave the final presentation on Day 1, which was an overview of Web Analytics. Garry started by identifying key similarities and differences between Web Analytics and Marketing Analytics, such as the differences in data sources. He went on to explain how Web Analytics is now the base for two separate disciplines - multi-channel CRM and online Conversion Rate Optimization. Garry demonstrated the power of the approaches by means of examples from several RedEye clients.

\section{Day 2 - Advanced analytics for the online world}

The second day was chaired by Edouard Servan-Schreiber (Teradata), who opened the proceedings with a presentation on social and multichannel marketing. Edouard stressed the separate viewpoints that different channels provide, and the importance of creating integrated data to support multi-channel marketing.

Mark Patron (RedEye) drilled deeper into some of the trends and issues in online Marketing Analytics. He included recent research results, which showed that companies are increasingly using behavioural segmentation for targeting emails and customer analysis. Mark discussed the critical importance of multi-channel marketing, with a case study on Monarch Airlines, and concluded with thoughts on alternative options for data integration.

Mark Rogers (Market Sentinel) discussed the use of Text Analytics for finding patterns in large textual databases, and Sentiment Analysis for tracking positive and negative feelings, particularly to identify trends and changes over time. Mark illustrated his presentation with numerous use cases and case study examples. 


\section{Social graphs}

\section{Respecting privacy}

Behavioural targeting

\section{Segmentation in the online world}

\section{Engagement scoring}

\section{Multivariate testing}

\section{Targeting anonymous web users}

Alpesh Doshi (Fintricity) focused on the Social Graph — the map of relationships between people that sits at the heart of SNA. He explained that capturing additional attributes — such as interests, actions, interactions and sentiment - can make a social graph more useful. Alpesh summarized the main applications of social graphs, including 'boosting' recommendation engines, understanding influence and segmenting users.

Dave Evans (Information Commissioner's Office) discussed the revised legislation on the storage and use of cookies, which comes into force in May 2012. Dave explained that the major web browsers can help, in terms of managing consent to cookies, but that companies should be taking a proactive approach — he gave the ICO's latest advice on this and summarized what the next steps will be.

Stuart Colman (AudienceScience) began by showing that online display advertising is booming, and that a key driver is audience targeting using behavioural data. Behavioural targeting uses multiple data sources (both online and off-line) to define an audience, and therefore can be more powerful than traditional methods of targeting. Data Management Platforms play a role in linking data sources together and defining behavioural segments. Finally, Stuart commented that data privacy is critical, and that the best industry response is selfregulation.

Neil Mason (Global Dawn) first explained the reasons for moving away from averages, when measuring web behaviour, and introduced the main types of behavioural segmentation - together with the process for developing a discovery-based segmentation using visitor profile data. Neil used a case study on royalmail.com to illustrate a behavioural segmentation.

Andrew Hood (Lynchpin Analytics) defined Online Engagement as 'Online visitor interactions with potential future value' - therefore, Engagement is a critical stage before Conversion. Andrew explained the different alternative measures of Engagement, and that the approaches with greatest business value tend to be more difficult to measure - the most useful being visitor-level participation.

Joe Doveton (GlobalMaxer) discussed the importance of multivariate testing as a tool for improving conversion rates. Joe then explained how this works at his company, including the use of a Cultural Database in order to build in an understanding of the ways in which different cultures will respond to different types of design. $\mathrm{He}$ presented a large number of client case studies to illustrate the impact of improving page design.

In contrast with behavioural targeting, which defines an audience of known email addresses, this presentation focused on anonymous web users who have not supplied any personal information. Malcolm Duckett (MAGIQ) showed that a considerable amount is known about anonymous users, including environmental information (eg IP addresses) and browsing behaviour, thus enabling relevant content and marketing messages to be served up. He explained that huge volumes of data have to be tracked; however, trends such as cloud computing 


\section{Attribution of media effects}

Distinguished authors and Software as a Service are bringing the costs down. Malcolm presented a series of case studies showing practical ideas that work.

Matthew Tod (Logan Tod) first explained the challenges currently being faced to understand how different campaigns impact consumer behaviour. He recommended that it is more practical to construct data on 'islands of excellence' that may be joined together, rather than attempting to produce a 'single view of customer'. Finally, Matthew proposed a four-phase attribution framework: Discovery, Attribution, Testing and Evaluation.

After two presentation-packed days, the conference closed with a panel session that actually overran - firm evidence, indeed, of the raft of ideas and thoughts that these sessions had provoked.

Barry Leventhal F IDM

\section{Practitioner book review: Spending advertising money in the digital age - how to navigate the media flow}

Hamish Pringle and Jim Marshall

Kogan Page, 2011; £26.39 (from Amazon); 316pp.

ISBN: 978-0-7494-6305-2 (Paperback)

Journal of Direct, Data and Digital Marketing Practice (2012) 14, 80-83. doi:10.1057/dddmp.2012.13

If anyone is qualified to write a book with this title, these two gentlemen are.

Hamish Pringle was until recently the Director General of the IPA (Institute Of Practitioners in Advertising) in the UK.

Jim Marshall is the Chief Client Officer at Aegis Media and is exMedia Director at Young and Rubicam, with a 35-year track record working in media at top agencies.

So what have these two media experts produced for us?

As a non-media specialist, I must admit that I approached doing his review (and in particular reading this book) with some trepidation. I need not have worried. I'm pleased to be able to report that this book is extremely easy to read. It is largely free of media-specific jargon. Terms are explained as they are introduced. Moreover, the book contains much insightful analysis of the current media scene and some thought-provoking ideas about where it's going. It contains many photographs (most taken by Hamish Pringle) and even some cartoons (as is explained, they are intended to 'lighten up' the chapter on Media Strategy).

The title is a homage to the classic 'Spending Advertising Money' written by Simon Broadbent in 1970. This new book seeks, among 